\section{Far-lateral approach with the "transverse-S" skin incision and conservative muscle dissection: a pragmatic surgical route}

TO THE EDITOR: We read with interest the article by Moscovici et al. ${ }^{4}$ (Moscovici S, Umansky F, Spektor S: "Lazy" far-lateral approach to the anterior foramen magnum and lower clivus. Neurosurg Focus 38(4):E14, April 2015).

Since the introduction of the far-lateral approach (FLA), ${ }^{1,3}$ several modifications and nuances regarding skin incision, muscle dissection, and degree of bone resection have been described in the literature.

The reality is that despite the evolution of microsurgical and skull base surgery techniques, approaching the lower clivus and anterior foramen magnum still poses a great challenge. The complex anatomy of the region requires a careful and time-consuming dissection in order to perform the FLA. Additionally, the approach by itself has associated morbidity that might result in complications such as arterial and venous injury, pseudomeningocele formation, CSF leakage, neural tissue injury, craniovertebral junction instability, and muscular atrophy. Nevertheless, we have wondered if a more conservative soft-tissue dissection and tailored bone removal could minimize the complications associated with the approach.

In this context, we read with great interest the recent paper by Moscovici et al. ${ }^{4}$ In this clinical study, the authors presented a modification of the FLA technique ("lazy" FLA) emphasizing the management of the vertebral artery (VA). The description of the technique is followed by their clinical experience showing excellent results in the treatment of 44 patients with tumors or vascular lesions.

The approach described consists of a lazy-S skin incision, beginning in the retroauricular area, descending vertically through the midpoint between the inion and mastoid, and curving in its lower part to the midline of a spinal process between C-4 and C-7. The splenius capitis and semispinalis capitis muscles are transected longitudinally and retracted to the side. The semispinalis capitis muscle is detached from the superior nuchal line, and beneath the superficial muscular layer, the fat pad is bluntly dissected to open the deep muscular layer that forms the suboccipital triangle. The rectus capitis major muscle is detached from the inferior nuchal line and taken medially; the superior oblique muscle is transected carefully in the middle.

The authors highlighted the technique of VA manipulation, advocated by the team at Lariboisière, ${ }^{2}$ that consists of preserving the periosteal layer and the venous plexus located between the periosteum and the VA. This maneuver enables elevation and partial mobilization of the horizontal segment of the VA between the transverse process/ vertebral foramen of C-1 laterally and the point of VA penetration into the atlantooccipital membrane medially. It facilitates hemilaminectomy of $\mathrm{C}-1$, providing enough room to reflect the VA caudally while still encased in its periosteal sheath.

We have used the same strategy to handle the VA and agree with the authors on the advantages of a tailored condyle bone resection, and preservation of the periosteal sheath and dural wrap when mobilizing the VA laterally. We also believe that such dissection is technically quicker to perform since there is no need to skeletonize the VA and to deal with its venous plexus. In addition, the softtissue cuff serves as a protection to the VA, minimizing the risk for vertebral injury while preserving a wide working space.

However, their lazy-S skin incision and layer-by-layer muscle dissection still resembles the classical descriptions. There is a wide variety of scalp incisions reported for FLA including the horseshoe (or U-shaped), inverted hockey stick (or L-shaped), C-shaped, and now the lazy-S incision. These incisions involve at least one of the following: large skin opening, sectioning through a muscle, and time-consuming muscle dissection in multiple layers. The consequences are prolonged dissection time, muscle bleeding, dead space in different muscle layers, postoperative neck pain, increased risk of pseudomeningocele and CSF leak, and muscle atrophy.

We hypothesized that a more targeted incision over the lateral aspect of the inferolateral skull base, offering the same degree of exposure while reducing the amount of muscle dissection, could minimize the risk of the aforementioned complications. A linear incision would provide a direct route, minimizing the dead space, but it would still require sectioning of several muscles and would not provide adequate anatomical exposure. We have also used the more conservative C-shaped incision and believe it is a good alternative, but the approach still involves multiple layers of muscle dissection and, in our experience, leads to significant postoperative muscle atrophy. 

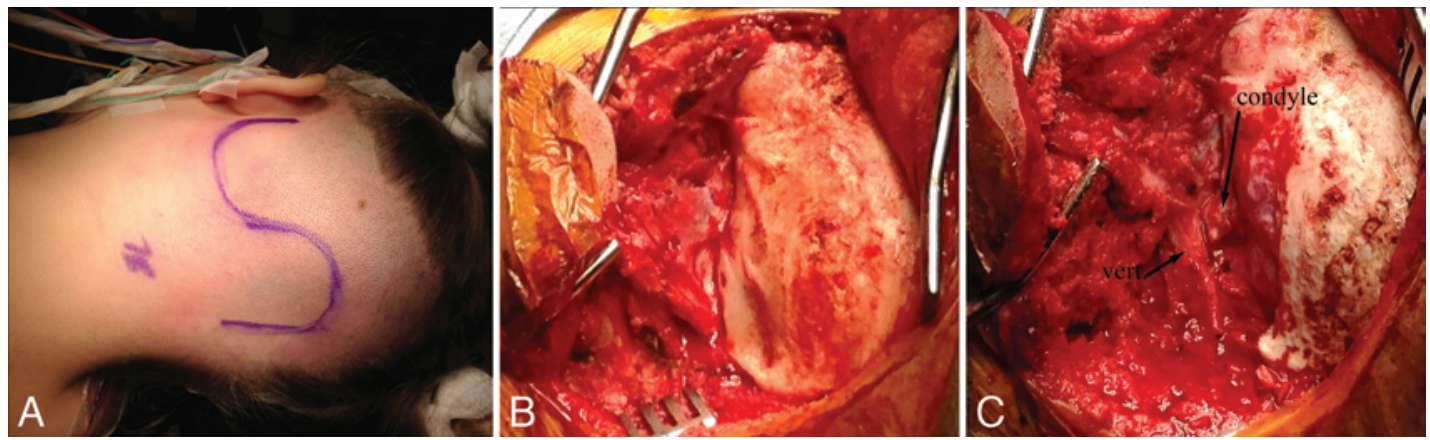

FIG. 1. A: Left-side transverse-S skin incision. B: Subperiosteal detachment of the muscles in a single layer from the superior nuchal. C: Exposure of the area of interest is not compromised by the skin incision or muscle dissection. Condyle $=$ occipital condyle; vert $=$ VA.

In the pursuit of improving patient's quality of life and postoperative results, we have changed our FLA strategy to a pragmatic transverse-S-shaped incision with conservative muscle dissection (Fig. 1). The scalp incision starts at the retroauricular area below the asterion, descending vertically and medially towards the first bend of the "S"; progresses superiorly and medially towards the second bend of the "S" that is located inferior to the superior nuchal line; and the incision is completed in a vertical descending trajectory in the midline to the level of the C-2 spinal process. Subcutaneous dissection is performed to provide sufficient exposure of the superior nuchal line. There are no muscle transections. The ipsilateral splenius capitis, semispinalis capitis, trapezius, and the sternocleidomastoid muscles are detached from the superior nuchal line and continued to the midline and inferiorly in a subperiosteal fashion. A small cuff of muscle insertion is preserved for later re-attachment of the muscle. Dissection is continued in the midline over the avascular plane of the nuchal ligament until the posterior arch of C-1 can be identified. The initial superior exposure is connected in the midline to the exposure of $\mathrm{C}-1$ in a subperiosteal manner, below the plane of the suboccipital triangle muscles. In that way, the rectus capitis posterior minor is detached from the inferior nuchal line and C-1 and the rectus capitis posterior major and oblique superior muscles are detached from the inferior nuchal line. All muscles are dissected in a single plane and retracted laterally and inferiorly. The transverse-S skin incision provides enough space for muscle retraction and bone exposure.

The progression of the lateral dissection is performed under the microscope to avoid VA injury. As described previously, we perform a dissection mobilizing the periosteal sheath of C-1 toward the VA and without infraction of the venous plexus; this is followed by a hemilaminectomy of C-1, tailored suboccipital craniectomy, and resection of the occipital condyle depending on the surgical corridor needed. If additional VA transposition is required, we proceed with lateral dissection over C-1 until the vertebral foramen is reached and opened. If additional inferior exposure is needed, we proceed with detachment and lateral displacement of the rectus capitis posterior major, oblique inferior, and semispinalis cervicis muscles of the C-2 spinal process.

The first few cases of craniovertebral junction tumors in which we have used this approach were promising. Our impression is that time to perform the FLA was significantly reduced; surgical exposure was not compromised by the muscle dissection; there were no complications related to the approach; and we observed no postoperative muscle atrophy. Further evaluation on the advantages of the transverse-S FLA over the other muscle-dissection strategies is still needed and will likely be performed as our case series increase.

In conclusion, we are enthusiasts of the subject and agree with Moscovici et al. ${ }^{4}$ on many surgical strategies to approach the craniovertebral junction. Yet we believe there is still room for improvement regarding soft-tissue transgression in FLA. We applaud the authors for their valuable contribution to the literature and their stimulating work in the field of skull base surgery.

André Beer-Furlan, MD

Daniel S. Ikeda, MD

Russell R. Lonser, MD

Daniel M. Prevedello, MD

Wexner Medical Center, The Ohio State University College of Medicine, Columbus, $\mathrm{OH}$

\section{Acknowledgment}

We would like to thank Dr. Caetano Coimbra (Dallas, TX) for his contribution on the development of this technique.

\section{References}

1. George B, Dematons C, Cophignon J: Lateral approach to the anterior portion of the foramen magnum. Application to surgical removal of 14 benign tumors: technical note. Surg Neurol 29:484-490, 1988

2. George B, Lot G: Anterolateral and posterolateral approaches to the foramen magnum: technical description and experience from 97 cases. Skull Base Surg 5:9-19, 1995

3. Heros RC: Lateral suboccipital approach for vertebral and vertebrobasilar artery lesions. J Neurosurg 64:559-562, 1986

4. Moscovici S, Umansky F, Spektor S: "Lazy" far-lateral approach to the anterior foramen magnum and lower clivus. Neurosurg Focus 38(4):E14, 2015

\section{Disclosures}

The authors report no conflict of interest. 


\section{Response}

We very much appreciate the comments of Dr. André Beer-Furlan and colleagues and read with great interest their technique to approach craniovertebral junction tumors. The authors describe their technique as a far-lateral approach using a "transverse-S" skin incision and conservative muscle dissection.

We have no experience with the use of a transverse incision in the posterior fossa or craniovertebral junction. In the past, we used the classic hockey stick skin incision. One step with this variation of the far-lateral approach was stripping the cervical muscles away from the squamous part of the occipital bone in an oblique-transverse direction and pulling them lateral and inferior. We abandoned this technique, as in our experience it was associated with a higher rate of wound-related complications such as pseudomeningocele formation, CSF leakage, and skin-edge necrosis. This motivated us to adopt our proposed lazy-S skin incision, with which we have had excellent results. In our experience, anatomical dissection through the muscle layers permits close approximation during wound closure. The muscles are reconnected in a more compact way; thus, no dead space remains, reducing the risk of a CSF leak and pseudomeningocele formation.

Another advantage of the longitudinal as opposed to the transverse incision is the ability to access large tumors that descend well below the foramen magnum and the C-1, $\mathrm{C}-2$, and even C-3 level.

We congratulate Beer-Furlan et al. on an interesting idea and promising preliminary results, and we would be interested to see their experience if they accumulate a larger series of cases in which they use their modification of the far-lateral approach.

Sergey Spektor, MD, PhD

Samuel Moscovici, MD

Felix Umansky, MD

Hadassah-Hebrew University Medical Center, Jerusalem, Israel

INCLUDE WHEN CITING

DOI: 10.3171/2015.9.FOCUS15480.

CAANS, 2016 